\title{
Scattering of Spin Polarized Positrons from Heavy Atoms: Europium and Bismuth
}

\author{
KAPIL K. SHARMA* \\ Department of Physics, Krishna Institute of Engineering and Technology, Ghaziabad (U.P.), India
}

(Received July 24, 2013; in final form June 2, 2014)

We present calculations of differential, integrated elastic, total momentum transfer cross-sections and spin polarization parameters $S$ for scattering of positron from $\mathrm{Eu}$ and $\mathrm{Bi}$ atoms in the energy range 2.0 to $500.0 \mathrm{eV}$ using relativistic approach. The target projectile interaction is represented both by real and complex parameter-free optical potentials in the solution of the Dirac equation for the scattered positrons. The theoretical results are obtained from relativistic approach based on solving the Dirac equation using Hartree-Fock and Dirac-Fock wave functions to calculate cross-sections at all the energies considered.

DOI: 10.12693 /APhysPolA.126.688

PACS: $34.80 . \mathrm{Bm}, 34.80 . \mathrm{Uv}$

\section{Introduction}

Interaction between electron/positron and radicals are known to be one of the key drivers of the physics and chemistry of industrial plasmas [1]. Chemical models to allow for optimized process control of plasma rectors consequently depend on the availability of the positron collision cross-sections, which give a quantitative understanding of positron interaction with given target, specially for the atomic and molecular radicals that are present in plasma. Also positron scattering have gained significant importance because the positron being a positively-charged probe, offers a more sensitive test of our ability to understand atomic interactions than the electron does. Additional importances of positron scattering derive from the fact that it involves interactions of matter with antimatter which have possible applications in the astrophysical arena. Despite the importance of such data, quantitative studies of collision cross-section with positrons are currently not widely available in the literature $[2,3]$. In our previous publication we reported differential cross-sections and spin polarization parameter for positron scattering by radon, radium, and gold atoms $[4,5]$ by optical potential approach.

A method which has been quite successful for calculating the scattering cross-sections is the model potential approach which seeks to use a complex local potential, generally represented by analytical expressions, to model the actual projectile-target interaction. The imaginary part of the interaction potential, $V_{\text {abs }}$, which is nonzero for projectile energies above the lowest inelastic threshold, accounts for the absorption effect, that is, absorption of incident projectile flux from the purely elastic channel. For positron scattering the real part of the interaction potential is partitioned into the static and correlation-polarization parts. The imaginary parts take into account various inelastic processes such as positronium formation as well as excitation and ionization of

\footnotetext{
*e-mail: kapil81_physics@yahoo.co.in
}

the target by positron impact. In the past, models of various parts of the total interaction potential have been proposed which contain adjustable parameters that are fitted to provide theoretical cross-sections. We focus on obtaining model interaction potentials which are free of any adjustable parameters. To the best of our knowledge, no such collision cross-sections for elastic processes currently exist in literature. As an alternative to electronatom scattering, both the similarities and the differences between electrons and positrons mean that positron scattering provide a useful, and sometimes more sensitive, test of the techniques used to study the electron and positron scattering processes.

The similarities between electrons and positrons mass, magnitude of charge, and spin suggest that a consistent approach to devising model potentials should incorporate these quantities using similar logic for both projectiles. The differences between electrons and positrons: the sign of the charge, the possibility of positronium formation, and the fact that positron projectiles are distinguishable from the electrons of the target atom while electron projectiles are not, offer important tests of how a model potential scheme handles issues such as projectile charge, inelastic thresholds, and correlations among projectile and target electrons. It is well known that positron-atom collisions can be described by many theoretical approaches and that is why it is important to test the various approximations with experimental measurements. In the present paper, we use the model potential approach to calculate the differential, intergraded elastic and total cross-sections, for positron scattering collisions with open shell elements like Eu and $\mathrm{Bi}$ atoms at $2.0 \mathrm{eV}$ to $500.0 \mathrm{eV}$ impact energies. The parameter-free model potentials that we employ in the present calculations are those that we have previously used, with good results, for positron scattering from radon, radium and gold atoms $[4,5]$. In view of this, the present model can be easily applied to study the elastic scattering from systems with open shell atoms fulfilling these requirements. 


\section{Theory}

\subsection{Interaction potentials}

In the present calculations, we employ a model potential of the positron-target system by a complex interaction potential $V(r)$ that consists of only three parts. These parts are the static potential $V_{\text {st }}(r)$, the correlation-polarization potential $V_{\mathrm{CP}}(r)$ and the absorption potential $V_{\text {abs }}(r)$, such that

$$
V(r)=V_{\mathrm{st}}(r)+V_{\mathrm{CP}}(r)+V_{\mathrm{abs}}(r) .
$$

\subsubsection{The static potential}

The static potential is the average over the ground state atomic charge distribution of the electrostatic interaction of the positron and atom. In case of positron, the static potential is repulsive in nature whereas it is attractive for electron scattering. It is determined by the radial part of the electron charge density of the target atoms, $\rho(r)$, which is obtained using both the non-relativistic atomic orbitals, from the Hartree-Fock wave function [6] for europium $(\mathrm{Eu})$ and bismuth $(\mathrm{Bi})$. The static potential, in atomic units, is given by

$$
V_{\mathrm{st}}(r)=\frac{Z}{r}-4 \pi \int \frac{\rho\left(r^{\prime}\right)}{r_{>}} r^{\prime 2} \mathrm{~d} r^{\prime},
$$

where $Z$ is the atomic number of the target atom and $r_{>}$ is the greater of $r$ and $r^{\prime}$.

\subsubsection{Positron correlation polarization potential}

The positron correlation polarization (PCP) potential is defined as a functional derivative of the corrective of the correlation energy with respect to $\rho(r)$ i.e.

$$
V_{\text {corr }}(r)=\left(1-\frac{1}{3} r_{\mathrm{s}} \frac{\mathrm{d}}{\mathrm{d} r_{\mathrm{s}}}\right) \varepsilon_{\text {corr }}\left(r_{\mathrm{s}}\right)
$$

with $r_{\mathrm{s}}$ as a density parameter satisfying $\frac{4}{3} \pi r_{\mathrm{s}}^{3} \rho(r)=1$, where $\rho(r)$ is the target undistorted electronic density. Finally, an analytic expression is obtained (in atomic units) $[7,8]$ as

$$
\begin{aligned}
& V_{\text {corr }}(r)= \\
& \left\{\begin{array}{l}
{\left[-1.82 / \sqrt{r_{\mathrm{s}}}+\left(0.051 \ln r_{\mathrm{s}}-0.115\right) \ln r_{\mathrm{s}}+1.167\right] / 2,} \\
\quad r_{\mathrm{s}}<0.302, \\
\left(-0.92305-0.09098 / r_{\mathrm{s}}^{2}\right) / 2, \quad 0.302 \leq r_{\mathrm{s}} \leq 0.56, \\
{\left[-8.7674 r_{\mathrm{s}}\left(r_{\mathrm{s}}+2.5\right)^{-3}+\left(-13.51+0.9552 r_{\mathrm{s}}\right)\right.} \\
\left.\quad \times\left(r_{\mathrm{s}}+2.5\right)^{-2}+2.8655\left(r_{\mathrm{s}}+2.5\right)^{-1}-0.6298\right] / 2, \\
\quad 0.56 \leq r_{\mathrm{s}} \leq 8.0 .
\end{array}\right.
\end{aligned}
$$

We further mention that in the limit $r_{\mathrm{s}} \rightarrow \propto$ the correlation polarization should approach the correct form of the polarization i.e. $V_{\mathrm{LR}}=-\alpha_{\mathrm{d}} / 2 r^{4}$, where $\alpha_{\mathrm{d}}$ is the dipole polarizability of the europium and bismuth atoms provided in Table I. Thus, depending on the location of the projectile from the target, $V_{\mathrm{PCP}}(r)$ for $\mathrm{e}^{+}-\mathrm{Eu}$ and $\mathrm{e}^{+}-\mathrm{Bi}$ atom system is taken as

$$
V_{\mathrm{PCP}}(r)= \begin{cases}V_{\text {corr }}(r), & r \leq r_{\mathrm{c}}, \\ V_{\mathrm{LR}}(r), & r \geq r_{\mathrm{c}} .\end{cases}
$$

Here $r_{\mathrm{c}}$ is the crossing point where $V_{\text {corr }}$ and $V_{\mathrm{LR}}$ cross to each other for the first time.

Electronic configuration, term symbols, dipole polarizability, ionization potential (I.P.),

first excitation threshold $E_{\mathrm{th}}$ and crossing points $\left(r_{\mathrm{c}}\right)$ and for Eu and Bi atoms.

\begin{tabular}{c|c|c|c|c|c|c|c}
\hline \hline $\begin{array}{c}\mathrm{Z} \\
\text { (atomic number) }\end{array}$ & Element & $\begin{array}{c}\text { Electronic } \\
\text { configuration }\end{array}$ & Term & $\begin{array}{c}\text { Polarizability } \\
{[\mathrm{a} . \mathrm{u} .]}\end{array}$ & $\begin{array}{c}\text { I.P. } \\
{[\mathrm{eV}]}\end{array}$ & $\begin{array}{c}E_{\mathrm{th}} \\
{[\mathrm{eV}]}\end{array}$ & $\begin{array}{c}\text { Crossing point } \\
{[\mathrm{a} . \mathrm{u} .]}\end{array}$ \\
\hline 64 & $\mathrm{Eu}$ & {$\left[X_{\mathrm{e}}\right] 6 s(2) 4 f(7)$} & ${ }^{8} S$ & 187.162 & 5.670 & 1.602 & 4.381 \\
83 & $\mathrm{Bi}$ & {$\left[X_{\mathrm{e}}\right] 6 s(2) 4 f(14) 5 d(10) 6 p(3)$} & ${ }^{4} S$ & 50.017 & 7.289 & 1.416 & 2.931 \\
\cline { 6 - 8 }
\end{tabular}

\subsubsection{Positron absorption potential}

The absorption potential, $V_{\text {abs }}$, which is non-zero for projectile energies above the lowest inelastic threshold, accounts for the absorption effects, i.e. absorption of incident projectile flux from the purely elastic channel. The absorption potential takes into account various inelastic processes such as positronium formation as well as excitation and ionization of the target by positron impact $[7,8]$. In the past, models of various parts of the total interaction potential have been proposed, which contain adjustable parameters that are fitted to provide theoretical cross-sections and match the experimental results. In our work, we focus on obtaining model interaction potentials that are free of any adjustable parameters.

According to the quasi-free scattering approximation, the absorption potential for a projectile with local kinetic energy $E=p^{2} / 2 m$ passing through a free electron gas of density $p(r)$ is given by $[7,8]$ :

$$
V_{\text {abs }}(r, E)=-\frac{1}{2} \rho(r) \bar{\sigma}\left(k_{\mathrm{F}}, p\right) v_{\mathrm{loc}} .
$$

Here, $v_{\text {loc }}=\left[2\left(E-V_{\mathrm{R}}(r)\right) / m\right]^{1 / 2}$ is the local velocity of the projectile for $\left(E-V_{\mathrm{R}}\right) \geq 0$ and $k_{\mathrm{F}}=\left[3 \pi^{2} \rho(r)\right]^{1 / 3}$ is the Fermi momentum. The $\bar{\sigma}\left(k_{\mathrm{F}}, p\right)$, the average quasi-free binary collision cross-section, is given as

$$
\begin{aligned}
\bar{\sigma}_{\mathrm{b}} & =\frac{1}{p} \int N\left(k_{\mathrm{F}}, q\right)|\boldsymbol{p}-\boldsymbol{q}| \mathrm{d} \boldsymbol{q} \\
& \times \int \frac{\mathrm{d} \sigma_{\mathrm{b}}}{\mathrm{d} \Omega}\left(\frac{1}{p_{0}^{2}} \delta\left(p_{0}-p_{\mathrm{f}}\right) \Theta\left(q^{\prime}, k_{\mathrm{F}}\right)\right) \mathrm{d} \boldsymbol{g} .
\end{aligned}
$$

Here, $\boldsymbol{p}\left(\boldsymbol{p}^{\prime}\right)$ and $\boldsymbol{q}\left(\boldsymbol{q}^{\prime}\right)$ are the laboratory frame momenta of the incident positron and target electron, respectively before and after the collision. The vectors $\boldsymbol{p}_{0}$ and $\boldsymbol{p}_{\mathrm{f}}$ are the initial and final momenta of the positron in center-of-mass frame of the binary system. The function $N\left(k_{\mathrm{F}}, q\right)$ refers to the target electron momentum distribution given as 


$$
N\left(k_{\mathrm{F}}, q\right)= \begin{cases}N\left(k_{\mathrm{F}}\right), & q \leq k_{\mathrm{F}}, \\ 0, & q>k_{\mathrm{F}}\end{cases}
$$

where $N\left(k_{\mathrm{F}}\right)=\frac{3}{4 \pi k_{\mathrm{F}}^{3}}$.

The momentum transfer vector $\boldsymbol{g}$, is given as $\boldsymbol{g}=\boldsymbol{p}^{\prime}$ $\boldsymbol{p}=\boldsymbol{q}-\boldsymbol{q}^{\prime}$. The $\frac{\mathrm{d} \sigma_{\mathrm{b}}}{\mathrm{d} \Omega}$ is the differential binary crosssection based on the Rutherford scattering as

$$
\frac{\mathrm{d} \sigma_{\mathrm{b}}}{\mathrm{d} \Omega}=2 / g^{4} \text {. }
$$

The function $\Theta\left(\boldsymbol{q}^{\prime}, k_{\mathrm{F}}\right)$ in Eq. (6) is unity for the Pauli allowed final states of the binary collision and zero for the Pauli blocked final states in the binary collision. For positron quasi-free scattering this function becomes

$$
\Theta\left(q^{\prime}, k_{\mathrm{F}}\right)=H\left(q^{\prime 2}-k_{\mathrm{F}}-\omega\right),
$$

where $\omega=2 \Delta$, with $\Delta$ being the energy gap between the target ground-state energy and the final energy of the originally bound target electron and $H\left(q^{\prime 2}-k_{\mathrm{F}}^{2}-\omega\right)$ is the Heaviside unit step function, which equals to one when the argument is non-negative and zero otherwise.

The physical interpretation of $\Theta\left(q^{\prime}, k_{\mathrm{F}}\right)$ is that, for an inelastic process to occur, the final energy of the target electron $q^{2} / 2$ must exceed the Fermi energy $E_{\mathrm{F}}=k_{\mathrm{F}}^{2} / 2$ by at least the energy gap $\Delta$. Processes that would allow the electron to fall into a lower-energy state are forbidden due to the Pauli blocking. The above definition of the Pauli blocking function $\Theta$ differs from that used for electron scattering [8] in that here, for positron scattering, Pauli blocking restrictions are applied to the target electrons only and no such restrictions are placed on the projectile.

It is interesting to examine the role of the energy gap in the model for positron scattering as compared to its role in the quasi-free model for electron scattering. For incident electrons $\Delta$ acts both as the energy needed to transfer a target electron from the highest occupied ground-state orbital to the first excited orbital and as the non-zero threshold for inelastic scattering. However, for incident positrons this situation is complicated since the formation of positronium (Ps) introduces another bound system, the binding energy of which can reduce the inelastic threshold to energy below the threshold for excitation $E_{\text {excit }}$. In fact, the quasi free absorption potential gives infinite cross-sections as $\Delta$ approaches zero. In our calculations of positron scattering from radon and radium atoms, we used for $\Delta$ the non-zero positronium formation threshold $E_{\mathrm{Ps}}$. The average binary collision cross-section $\bar{\sigma}_{\mathrm{b}}$ can be expressed as

$$
\begin{aligned}
& \bar{\sigma}_{\mathrm{b}}\left(k_{\mathrm{F}}, p\right)=\frac{16 \pi^{2}}{p^{2}} N\left(k_{\mathrm{F}}\right) \\
& \quad \times\left\{\begin{array}{l}
\frac{4}{3} \frac{k_{\mathrm{F}}^{3}}{\omega}+4 k_{\mathrm{F}}+2 p \ln \left|\frac{p-k_{\mathrm{F}}}{p+k_{\mathrm{F}}}\right|, \quad p^{2}-\omega \geq k_{\mathrm{F}}^{2}, \\
\frac{4}{3} \frac{\left(p^{2}-\omega\right)^{3 / 2}}{\omega}+4\left(p^{2}-\omega\right)^{1 / 2}+2 p \ln \left|\frac{p-\sqrt{p^{2}-\omega}}{p+\sqrt{p^{2}-\omega}}\right|, \\
k_{\mathrm{F}}^{2} \geq p^{2}-\omega \geq 0 .
\end{array}\right.
\end{aligned}
$$

2.2. Cross-section and spin polarization

The theoretical methodology concerning the mathematical formulation of positron-atom scattering has been discussed in [4]. So only a brief outline of the theory will be given here. The motion of the projectile positron in a central field $V(r)$ is described by the Dirac equation.

The motion of the projectile positron in a central field $V(r)$ is described by the Dirac equation

$$
\left[c \boldsymbol{\alpha} \cdot \boldsymbol{p}+\beta m_{0} c^{2}-V(r)\right] \psi=E \psi \text {. }
$$

For central potential, the Dirac equation can be reduced to a set of two equations

$$
g_{\ell}^{ \pm^{\prime \prime}}+\left[K^{2}-\frac{\ell(\ell+1)}{r^{2}}-U_{\ell}^{ \pm}(r)\right] g_{\ell}^{ \pm}(r)=0,
$$

where $g_{\ell}^{ \pm}$is related to the radial part $G_{\ell}^{ \pm}$of the large component of $\Psi$ as

$$
\begin{aligned}
G_{\ell} & =\sqrt{\eta} \frac{g_{\ell}}{r}, \quad \eta=\frac{\left[E-V(r)+m_{0} c^{2}\right]}{c \hbar}, \\
K^{2} & =\frac{E^{2}-m_{0}^{2} c^{4}}{c^{2} \hbar^{2}} .
\end{aligned}
$$

Here, we take the total energy of the incident particle as $E=m_{0} \gamma c^{2}=E_{\mathrm{i}}+m_{0} c^{2}, \gamma=\left(1-v^{2} / c^{2}\right)^{-1 / 2}$ where $E_{\mathrm{i}}$ is the kinetic energy of the incident particle of rest mass $m_{0}$ and velocity $v . \alpha$ and $\beta$ are the usual $4 \times 4$ Dirac matrices. $U_{\ell}^{ \pm}$are the effective Dirac potentials and are given in atomic units $\left(m_{0}=e=\hbar=1,1 / c=\alpha\right.$, where $\alpha$ is fine structure constant) as

$$
\begin{aligned}
& -U_{\ell}^{ \pm}(r)=-2 \gamma V(r)+\alpha^{2} V^{2}(r)-\frac{3}{4} \frac{\left(\eta^{\prime}\right)^{2}}{\eta^{2}}+\frac{1}{2} \frac{\eta^{\prime \prime}}{\eta} \\
& \pm\left(\frac{\ell+1}{\ell}\right) \frac{1}{r} \frac{\eta^{\prime}}{\eta} .
\end{aligned}
$$

Here single and double primes denote the first and second derivatives with respect to $r$, respectively. It should be noted that the last term of $U_{\ell}^{ \pm}$in Eq. (13) corresponds to the two eigenvalues of the well-known spin-orbit interaction, one due to spin up and the other due to spin down

$$
\frac{1}{4 m_{0}^{2} c^{2}} \frac{1}{r} \frac{\mathrm{d} V(r)}{\mathrm{d} r} \boldsymbol{\sigma} \cdot \boldsymbol{L} .
$$

In the non-relativistic treatment of the Schrödinger equation, the above term is treated as a small perturbation along with the projectile target interaction. Here $\sigma$ is related to the spin $S$ as $\boldsymbol{\sigma}=2 S$ and the value of $\langle\boldsymbol{\sigma} \cdot \boldsymbol{L}\rangle$ equals for $j=(\ell+1 / 2)$ and $-(\ell+1)$ for $j=(\ell-1 / 2)$. The proper solution of Eq. (12) behaves asymptotically as

$$
\begin{aligned}
& g_{\ell}^{ \pm}(K, r) \sim K r\left[j_{\ell}(K r)-\tan \delta_{\ell}^{ \pm} \eta_{\ell}(K r)\right], \\
& \quad r \rightarrow \infty,
\end{aligned}
$$

where $j_{\ell}$ and $\eta_{\ell}$ are spherical Bessel functions of the first and second kind, respectively, and $\delta_{\ell}^{ \pm}$are the phase shifts due to collision interaction. The plus sign corresponds to the incident particles with spin-up and the minus sign in $\delta$ to those with spin-down. The phase shift $\delta_{\ell}^{ \pm}$can be obtained from the values of the radial wave function $g_{\ell}^{ \pm}$ at the two adjacent points $r$ and $(r+h)(h \ll r)$ at very large $r$ as

$$
\tan \delta_{\ell}^{ \pm} \approx-\frac{(r+h) g_{\ell}^{ \pm}(r) j_{\ell}[K(r+h)]-r g_{\ell}^{ \pm}(r+h) j_{\ell}(K r)}{r g_{\ell}^{ \pm}(r+h) \eta_{\ell}(K r)-(r+h) g_{\ell}^{ \pm}(r) \eta_{\ell}[K(r+h)]} .
$$


In the present calculation, the wave functions $g_{\ell}^{ \pm}$are obtained by numerical integration of Eq. (12) using Numerov's method. The two complex scattering amplitudes $f(K, \theta)$ (the direct amplitude) and $g(K, \theta)$ (the "spin-flip" amplitude) are defined as:

$$
\begin{aligned}
& f(K, \theta)=\frac{1}{2 \mathrm{i} K} \sum_{\ell=0}^{\infty}\left\{(\ell+1)\left[\exp \left(2 \mathrm{i} \delta_{\ell}^{+}\right)-1\right]\right. \\
& \left.\quad+\ell\left[\exp \left(2 \mathrm{i} \delta_{\ell}^{-}-1\right)\right]\right\} P_{\ell}(\cos \theta), \\
& g(K, \theta)=\frac{1}{2 \mathrm{i} K} \sum_{\ell=1}^{\infty}\left[\exp \left(2 \mathrm{i} \delta_{\ell}^{-}\right)-\exp \left(2 \mathrm{i} \delta_{\ell}^{+}\right)\right] \\
& \quad \times P_{\ell}^{1}(\cos \theta),
\end{aligned}
$$

where $\theta$ is the scattering angle and $P_{\ell}(\cos \theta)$ and $P_{\ell}^{1}(\cos \theta)$ are the Legendre polynomial and the Legendre associated functions, respectively. The elastic differential cross-section for scattering of the unpolarized incident electrons beam is given by

$$
\sigma(\theta)=\frac{\mathrm{d} \sigma}{\mathrm{d} \Omega}=|f|^{2}+|g|^{2}
$$

and the spin polarization parameters $S(\theta), T(\theta)$ and $U(\theta)$ have the forms by

$$
\begin{aligned}
& S(\theta)=\frac{\mathrm{i}\left(f g^{*}-f^{*} g\right)}{\sigma(\theta)}, \quad T(\theta)=\frac{|f|^{2}-|g|^{2}}{\sigma(\theta)}, \\
& U(\theta)=\frac{f g^{*}+f^{*} g}{\sigma(\theta)} .
\end{aligned}
$$

The Sherman function $S$ describes the spin polarization of the scattered positrons if the incident positron beam is unpolarized. In this paper, large numbers of phase shift depending on the impact energy were evaluated using the Born approximation.

\section{Results and discussions}

\subsection{Differential cross-section and asymmetry parameters}

Now, we present our elastic DCS and $S$ parameter for positron scattering from Eu and Bi atom in Figs. 1 to 4 at energy range 10.0 to $500.0 \mathrm{eV}$. Here we have presented our results for both real and complex potentials. It is seen that the present theory predicts the forward peaks, number of minima and maxima at middle angle enhanced backward slope at the DCS. Our calculated DCS's with $p Q V_{\text {a }}$ absorption changes dramatically for $E>10.0 \mathrm{eV}$. The calculated angular variation exhibits deeper minima, which occur at slightly higher angles compared to the DCS in SP model employing real potentials. Furthermore, we have also computed the DCS for $\mathrm{e}^{+}-\mathrm{Eu}$ and $\mathrm{e}^{+}-\mathrm{Bi}$ scattering employing electron quasi-free absorption $p Q V_{\mathrm{a}}$ with the same static and polarization interaction as used with $p Q V_{\mathrm{a}}$. This would enable us to examine the effect of $p Q V_{\mathrm{a}}$ on DCS. In the same figure we also present spin-polarization parameter (the Sherman function) at the same energies for the atoms $\mathrm{Eu}$ and $\mathrm{Bi}$. At low energies, the present phenomenological absorption potential appears not to bring any changes in the calculated values, however at higher impact energies, the results are presented in the both models SP and SPa. In all cases, $S$-parameter exhibits a rapid variation with scattering angles. Our calculations show structure in the DCS curves between $20^{\circ}$ and $90^{\circ}$, which become more pronounced with increasing atomic number.

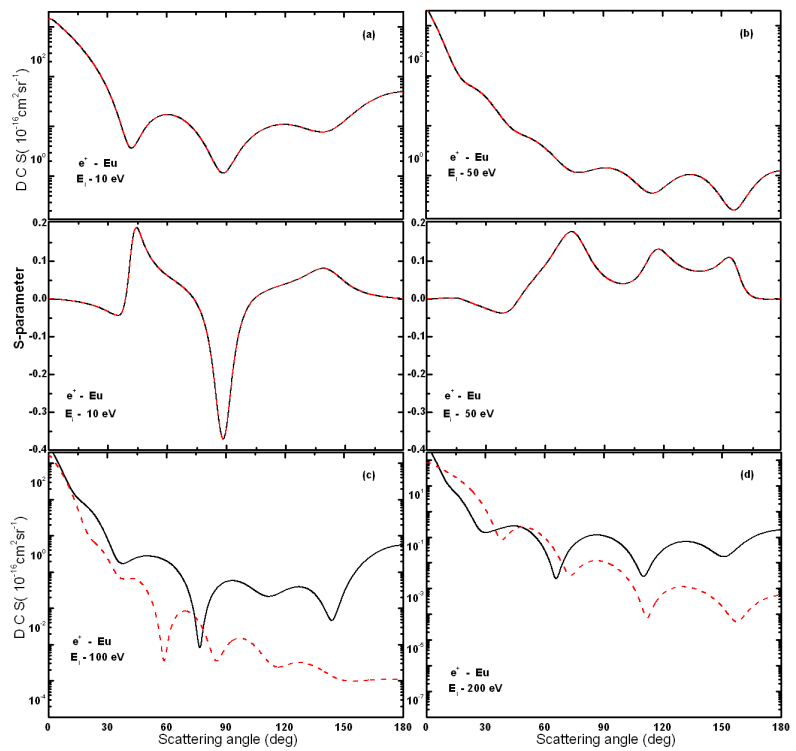

Fig. 1. DCS and $S$-parameter for elastic $\mathrm{e}^{+}-\mathrm{Eu}$ scattering at energies (a) $10 \mathrm{eV}$, (b) $50 \mathrm{eV}$, (c) $100 \mathrm{eV}$, (d) $200 \mathrm{eV}$. Present calculation: - with complex potential $(\mathrm{SPa}),-$ - - $^{-}$with real potential $(\mathrm{SP})$.

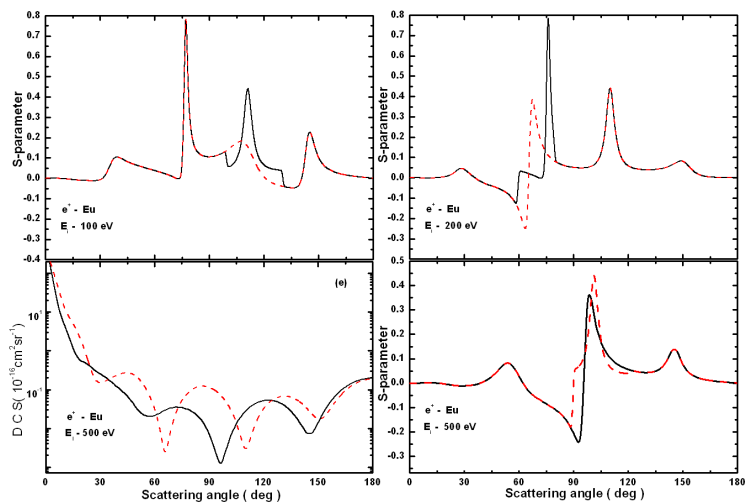

Fig. 2. DCS and $S$-parameter for elastic $\mathrm{e}^{+}-\mathrm{Eu}$ scattering at energies (e) $500 \mathrm{eV}$, Present calculation: with complex potential $(\mathrm{SPa})$; ---- with real potential (SP).

For both targets considered here DCS curves do not flatten at all scattering angles. While it is clear that this structure is due to diffraction effects, the precious physics at how and where the local minima arise in DCS at intermediate energies is not well understood to date. Recently, an attempt to explain such structure for electron scattering was done using semiclassical approach $[9,10]$. This semiclassical explanation, however, does not account for the structure seen in positron atom - differ- 


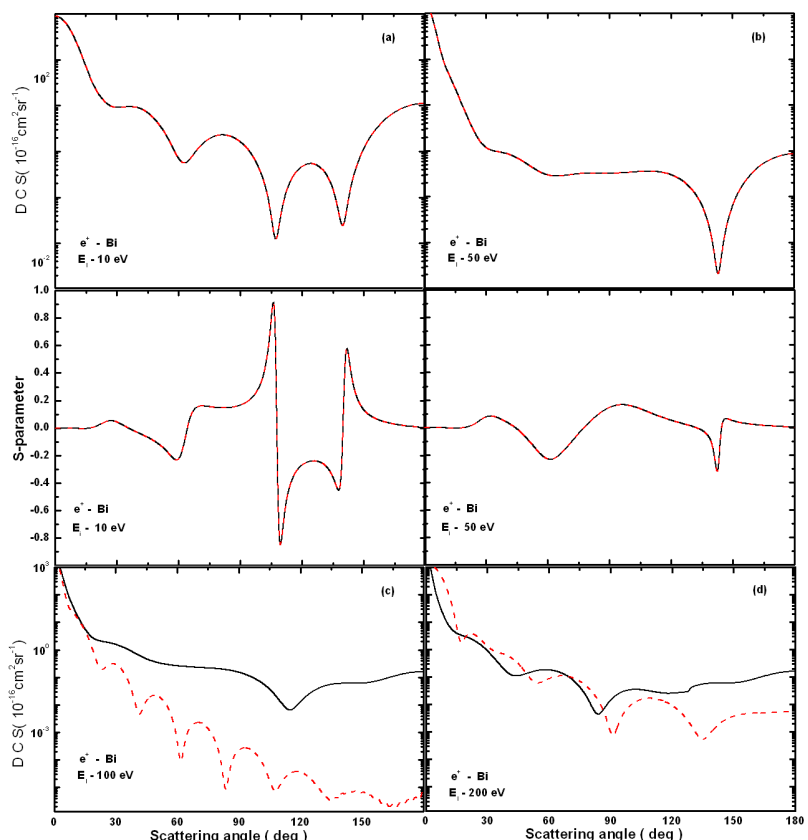

Fig. 3. DCS and $S$-parameter for elastic $\mathrm{e}^{+}-\mathrm{Bi}$ scattering at energies (a) $10 \mathrm{eV}$, (b) $50 \mathrm{eV}$, (c) $100 \mathrm{eV}$, (d) $200 \mathrm{eV}$. Present calculation: — with complex potential $(\mathrm{SPa})$; - - - - with real potential (SP).

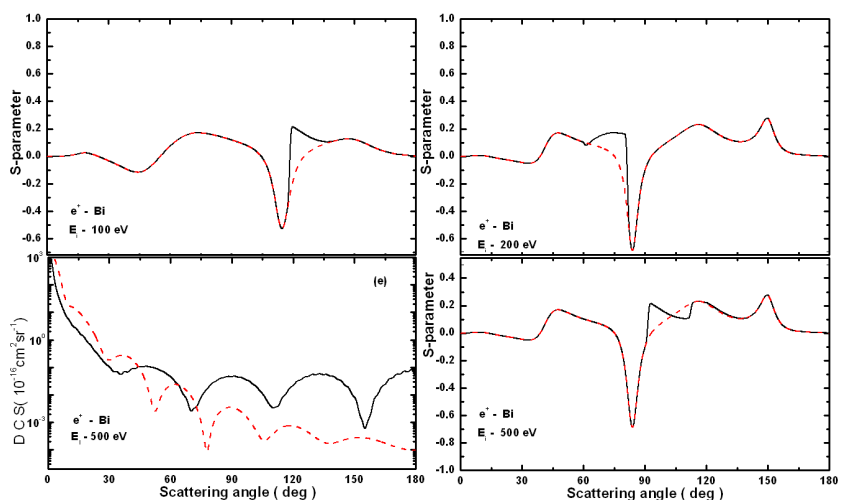

Fig. 4. DCS and $S$-parameter for elastic $\mathrm{e}^{+}$-Bi scattering at energies (e) $500 \mathrm{eV}$. Present calculation: — with complex potential (SPa); ---- with real potential (SP).

ential cross-sections. One of the most interesting aspects of this study is that the use of the quasifree absorption potential remains a viable option for positron scattering from heavier atoms despite the fact that the positronium formation channel for these systems is always open $[7,8]$.

We expect that there is a general explanation that accounts for the locations of the minima in both electron and positron scattering data; to data this is general explanation has not been worked out.

\subsection{Angle-integrated elastic cross sections and contribution of partial waves}

We have performed calculations in different models, which are abbreviated as follows: S, static only, SP, S plus, and polarizations potentials $\mathrm{SPa}$ the absorption potentials. In the present study we report our calculations $\mathrm{SP}$ and SPa model as obtained HF wave functions [6]. Let us discuss first our partial cross-sections for the $s, p$, $d$ and $f$ waves in SPa approximation for $\mathrm{Eu}$ and $\mathrm{Bi}$ as shown in Fig. 5a,b.

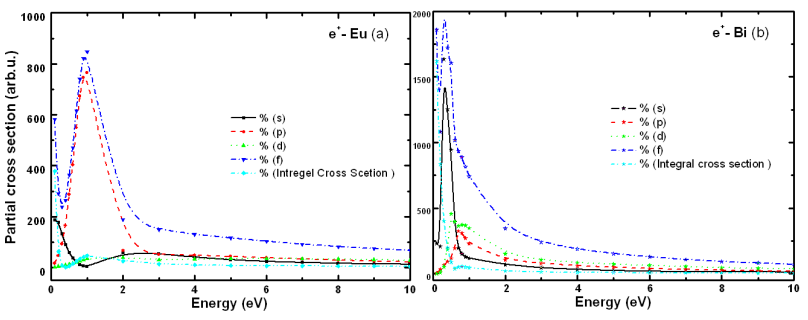

Fig. 5. (a)-(b) Partial cross-sections in unit of $10^{-16} \mathrm{~cm}^{2}$ for scattering of $\mathrm{e}^{+}-\mathrm{Eu}$ and $\mathrm{e}^{+}-\mathrm{Bi}$. Present calculation $s, p, d$, and $f$ waves, and summed integral cross-section.

For Eu atom as can be seen from Fig. 5 in low energy region $E_{\text {in }}<10 \mathrm{eV}$, the main contribution to the partial cross-section is from $f$ and $p$ wave. In the elastic region, the maximum of the cross-section comes from $f$ wave whereas near and beyond the inelastic threshold $p$ wave makes maximum contribution. The maximum $f$-waves cross-sections arise from the shape resonance at energies $E_{\mathrm{r}}$ equal to $1.1 \mathrm{eV}$ for $\mathrm{Eu}$ atom and $0.68 \mathrm{eV}$ for Bi atom. The total cross-sections are also plotted in the figures in this model. Each curve shows a narrow low energy maximum followed by sharp fall of the cross-section up to first inelastic threshold. Now turning our attention to $\mathrm{Bi}$ atoms as displayed in Fig. $5 \mathrm{~b}$ it is seen that the total cross-section shows maximum at low energy and then falls off smoothly with the increase in the impact energies. The broad structure is due to the maximum in each of these $p, d, f$, and $s$ partial cross-section.

\subsection{Elastic, total, and momentum-transfer cross-sections}

The results of our present integrated elastic, total, and momentum-transfer cross-sections for $\mathrm{Eu}$ and $\mathrm{Bi}$ atoms are presented in Tables II and III. The elastic cross-sections are obtained using both the real and complex potentials. It is noted that at all energy the elastic cross-sections $\left(\sigma^{\prime}\right)$ obtained with only real potential are larger than those obtained with the complex potential $(\sigma)$. This is similar to earlier observations with several other heavier atoms [11-14]. We have also shown in the tables the cross-section with only absorption potential $\left(\sigma_{\text {abs }}\right)$ as well as the total cross-section $\left(\sigma_{\mathrm{t}}\right)$ which includes all the elastic and inelastic cross-sections. The total cross-section descends rapidly at lower energies and thereafter varied slowly with increase impact energies. 
TABLE II

Elastic $\left(\sigma_{\mathrm{el}}\right)$, absorption $\left(\sigma_{\mathrm{abs}}\right)$, and total $\left(\sigma_{\mathrm{t}}\right)$ cross-sections in units of $10^{-16} \mathrm{~cm}^{2}$ for Eu and $\mathrm{Bi}$ in $\mathrm{SPa}$ with absorption effect. $\sigma_{\mathrm{el}}^{\prime}$ is the elastic scattering cross-section without absorption effect. Momentum transfer cross-section $\left(\sigma_{\mathrm{m}}\right)$ in units of $10^{-16} \mathrm{~cm}^{2}$ for $\mathrm{e}^{+}-\mathrm{Eu}$ and $\mathrm{e}^{+}-\mathrm{Bi}$ scattering.

\begin{tabular}{c|c|c|c|c|c|c|c|c}
\hline \hline \multirow{2}{*}{$\mathrm{E}$} & \multicolumn{3}{|c|}{$\sigma^{+}$-europium $(\mathrm{Eu})$} & \multicolumn{4}{c}{$\sigma^{+}$-bismuth $(\mathrm{Bi})$} \\
\cline { 2 - 9 }$[\mathrm{eV}]$ & $\sigma_{e \lambda}^{\prime}$ & $\sigma_{e \lambda}$ & $\sigma_{\mathrm{abs}}$ & $\sigma_{\mathrm{t}}$ & $\sigma_{e \lambda}^{\prime}$ & $\sigma_{e \lambda}$ & $\sigma_{\mathrm{abs}}$ & $\sigma_{\mathrm{t}}$ \\
\hline 2.0 & 679.2 & 679.1 & 0 & 679.1 & 856.5 & 856.6 & 0 & 856.5 \\
5.0 & 563.4 & 563.4 & 0 & 563.4 & 842.7 & 842.7 & 0 & 842.5 \\
10.0 & 405.2 & 399.4 & 7.23 & 406.6 & 799.3 & 799.3 & 0 & 799.7 \\
20.0 & 271.8 & 265.2 & 9.78 & 275.0 & 743.3 & 734.4 & 8.99 & 743.0 \\
30.0 & 223.6 & 210.6 & 12.98 & 223.6 & 627.6 & 623.9 & 9.76 & 632.8 \\
50.0 & 182.9 & 178.8 & 13.08 & 191.9 & 429.9 & 429.5 & 7.43 & 437.0 \\
80.0 & 151.5 & 135.0 & 15.31 & 150.5 & 373.7 & 367.8 & 9.12 & 377.0 \\
100.0 & 136.8 & 121.5 & 15.78 & 137.3 & 322.6 & 318.9 & 7.55 & 325.6 \\
150.0 & 110.3 & 104.9 & 6.9 & 111.9 & 245.1 & 234.8 & 10.88 & 244.9 \\
200.0 & 92.74 & 90.21 & 3.98 & 94.9 & 202.7 & 198.4 & 5.77 & 203.8 \\
250.0 & 80.42 & 72.92 & 7.09 & 80.01 & 174.5 & 170.8 & 6.22 & 177.0 \\
300.0 & 71.41 & 65.2 & 7.99 & 73.19 & 155.6 & 150.8 & 3.33 & 154.3 \\
350.0 & 64.59 & 59.12 & 8.08 & 67.20 & 140.6 & 138.9 & 2.99 & 141.1 \\
400.0 & 59.24 & 54.17 & 9.09 & 63.26 & 128.2 & 127.4 & 2.87 & 129.2 \\
450.0 & 54.93 & 50.22 & 5.07 & 55.29 & 118.8 & 115.8 & 2.12 & 118.1 \\
500.0 & 51.38 & 46.44 & 5.09 & 51.53 & 109.6 & 101.8 & 1.89 & 103.7
\end{tabular}

TABLE III

Momentum-transfer cross-section $\left(\sigma_{\mathrm{mt}}\right)\left(a_{0}^{2}\right)$ for positron scattering from $\mathrm{e}^{+}-\mathrm{Eu}$ and $\mathrm{e}^{+}-\mathrm{Bi}$ scattering.

\begin{tabular}{c|c|c|c|c}
\hline \hline \multirow{2}{*}{$\mathrm{E}[\mathrm{eV}]$} & \multicolumn{2}{|c|}{$\boldsymbol{\sigma}^{+}$-europium $(\mathrm{Eu})$} & \multicolumn{2}{c}{$\boldsymbol{\sigma}^{+}$-bismuth $(\mathrm{Bi})$} \\
\cline { 2 - 5 } & $\mathrm{SP}$ & $\mathrm{SPa}$ & $\mathrm{SP}$ & $\mathrm{SPa}$ \\
\hline 2.0 & 98.88 & 98.88 & 67.20 & 67.20 \\
5.0 & 87.07 & 87.17 & 47.03 & 47.07 \\
10.0 & 76.07 & 76.47 & 32.70 & 30.80 \\
20.0 & 57.39 & 57.09 & 21.55 & 21.24 \\
30.0 & 45.06 & 47.59 & 17.89 & 15.47 \\
50.0 & 4.80 & 4.98 & 12.27 & 9.40 \\
80.0 & 4.50 & 3.38 & 4.49 & 5.59 \\
100.0 & 2.53 & 2.72 & 3.20 & 4.38 \\
150.0 & 4.64 & 1.82 & 3.91 & 2.65 \\
200.0 & 3.54 & 1.34 & 2.14 & 1.87 \\
250.0 & 1.06 & 1.05 & 3.59 & 1.41 \\
300.0 & 1.85 & 0.87 & 1.07 & 1.11 \\
350.0 & 1.83 & 0.73 & 1.05 & 0.92 \\
400.0 & 1.99 & 0.62 & 1.26 & 0.78 \\
450.0 & 1.29 & 0.53 & 1.69 & 0.67 \\
500.0 & 1.71 & 0.47 & 1.19 & 0.59
\end{tabular}

\section{Conclusion}

In this paper, we have presented the result for DCS and the angular variation of spin polarization parameter $S$ for positrons, scattered from europium (Eu) and bismuth $(\mathrm{Bi})$ at energies between 2.0 and $500.0 \mathrm{eV}$. This calculation has been performed using two differential models - without and with absorption potential. The positron scattering, the present result shows that the relativistic effects are important and the spin polarization is hardly influenced by the spin-orbit coupling, which is consistent with earlier results. From these studies, we found that further research work is required for complete atomic behavior of europium ( $\mathrm{Eu})$ and bismuth (Bi) atoms. We have presented our relativistic theoretical results for the elastic integral, momentum transfer, total cross-section; DCS and the angular variation of spin polarization parameters for positron from $\mathrm{Eu}$ and $\mathrm{Bi}$ atom at energies 2.0 to $500.0 \mathrm{eV}$. We have performed the calculations in two models, the first one includes a parameter-free correlation polarization potential to account for the polarization of atomic charge cloud and the other one uses a phenomenological absorption potential to account for loss of electron flux into the nonelastic channels in addition to the polarization potential. Further, the positron scattering from heavy species presented here shows significant amount of spin polarization in the scattered beam at various scattering angles. The present calculations suggest that the consistent model potential can produce reliable total cross-sections for positron-atom scattering at higher impact energies. This clearly indicates that there is a need for experimental measurements and other theoretical calculations in this energy region, so that it may provide a possibility of assessing accuracy of the present optical model. We hope that new experimental results will be reported in the light of our calculations for the $\mathrm{e}^{+}-\mathrm{Eu}$ and $\mathrm{e}^{+}-\mathrm{Bi}$ scattering.

\section{Acknowledgments}

Author is thankful to the Director (Dr. Narendra Kumar) and HOD Applied science (Dr. C.M. Batra and Dr. Vipin Kumar) of the institute for their constant encouragement and supports.

\section{References}

[1] D.B. Graves, M.J. Kushner, J.W. Gallagher, A. Garscadden, G.S. Oerlein, A.V. Phelps, Database Needs for Modelling and Simulation of Plasma Processing, National Research Council, National Academy Press, Washington, DC 1996.

[2] N.J. Mason, J. Phys. D: Appl. Phys. 42, 194003 (2009).

[3] M.J. Kushner, Bull. Am. Phys. Soc. 55, 107 (2010).

[4] K.K. Sharma, R.P. Vats, Phys. Scr. 85, 045304 (2012).

[5] K.K. Sharma, Neerja, R.P. Vats, J. At. Mol. Sci. 2, 294 (2011).

[6] A.D. McLean, R.S. McLean, At. Data Nucl. Data Tables 26, 197 (1981).

[7] D.D. Reid, J.M. Wadehra, Phys. Rev. A 57, 2583 (1998).

[8] D.D. Reid, J.M. Wadehra, J. Phys. B, At. Mol. Opt. Phys. 29, L127 (1996).

[9] Neerja, A.N. Tripathi, A.K. Jain, Phys. Rev. A 61, 032713 (2000).

[10] Neerja, A.N. Tripathi, Eur. Phys. J. D 13, 5 (2001).

[11] G. Staszewska, D.W. Schwenka, D. Thirumamai, D.G. Truhlar, Phys. Rev. A 28, 2740 (1983).

[12] H. Mohan, A.K. Jain, S. Sharma, J. Phys. Conf. Ser. 199, 012023 (2012).

[13] R.K. Gangwar, A.N. Tripathi, L. Sharma, R. Srivastava, J. Phys. B 43, 085205 (2010).

[14] K.K. Sharma, Neerja, R.P. Vats, J. At. Mol. Sci. 3, 197 (2012). 